\title{
C1Q NEPHROPATHY AS A DIFFERENTIAL DIAGNOSIS OF LUPUS NEPHRITIS
}

Fernanda Maria da Silva Bezerra1,, , Tayana Evelling Uchôa da Silva ${ }^{1}$, Bárbara Seabra Carneiro ${ }^{1}$, Luiz Fernando Souza Passos ${ }^{1}$

1. Hospital Universitário Getúlio Vargas, Manaus (AM), Brazil.

${ }^{\star}$ Corresponding author: fernandaa.bezerra@hotmail.com

\section{BACKGROUND}

The C1q nephropathy (C1qN) is a glomerulonephritis rare, characterized by an immunofluorescence (IF) pattern with complementary dominant or codominant Clq and electron-dense deposits in the mesangium, without clinical or serological characteristics of lupus nephritis. It usually presents in children and young adults with simple proteinuria or frank nephrotic syndrome, without extrarenal manifestations, being associated with a high proportion of nonresponse to corticosteroids. The most common histopathological findings of $\mathrm{ClqN}$ are focal segmental glomerulosclerosis (FSGS) and minimal injury.

\section{CASE REPORT}

A.P.A., 19 years old, previously healthy female, with a history of progressive edema, dyspnea at rest, alopecia, hypoalbuminemia, hypercholesterolemia, anti-dsDNA positive on a single occasion, anti-HIV, HBsAg and anti-HCV negative, normal complement, ANA negative in different dosages, $24 \mathrm{~h}$ proteinuria of $1.7 \mathrm{~g}$. Ultrasonography of kidneys and urinary tract with hyper-refringence and reduced echogenicity of the pyramids, preserved corticomedullary relationship and size. The histopathological study revealed segmental and focal proliferative glomerulonephritis, absence of chronic sclerotic-tubular interstitial changes and mild lymphomononuclear tubulointerstitial nephritis. Immunofluorescence was positive (dominant) for $\mathrm{Clq}(++)$, IgM and $\mathrm{C} 3(+)$, IgA and $\mathrm{C} 3$ cylinders, consistent with immune-mediated glomerulopathy. A patient who underwent pulse therapy with methylprednisolone and cyclophosphamide for 5 months, without satisfactory response to therapy, with worsening of edemigenic syndrome, reduced urinary volume, increased proteinuria to $7.6 \mathrm{~g} / 24 \mathrm{~h}$ and creatinine to $1.95 \mathrm{mg} / \mathrm{dL}$, was chosen to perform a second biopsy with result of collapsing FSGS, with predominance of $\mathrm{C} 1 \mathrm{q}$ and $\lg \mathrm{M}(++)$ in IF.

\section{CONCLUSION}

The $\mathrm{C} 1 \mathrm{qN}$ covers a variety of biopsy findings that share dominant $\mathrm{C} 1 \mathrm{q}$ deposition, with varied clinical presentation. Those with nephrotic syndrome generally have nonspecific light microscopy findings, with extensive erasure of the podocyte process and mesangial deposit demonstrated by electron microscopy, identified as $\mathrm{C} 1 \mathrm{q}$ by immunofluorescence. About $77 \%$ of them respond to immunosuppression. Patients with sclerosing or proliferative lesions revealed by light microscopy, have a worse prognosis. C1q deposits may also suggest lupus nephritis. In 1985, Jennette and Hipp described C1qN as a lesion "similar to lupus nephritis". However, the absence of dominant or codominant immunoglobulin $\mathrm{G}$ reticular deposits or aggregates and the lack of a history compatible with systemic lupus erythematosus rule out the diagnosis. Clinical findings, with negative lupus serology, optical microscopy with a segmental and focal proliferative glomerulonephritis pattern and IF with dominant $\mathrm{Clq}$ deposition suggest the diagnosis of $\mathrm{C} 1 \mathrm{gN}$, as well as the poor response to corticosteroid therapy, which is also useful for differentiate it from lupus nephritis, which is classically sensitive to them. 